\title{
Ying Chen et l'appel du risque
}

\author{
Lucie Lequin
}

Within the context of literary scholarship, argues Lucie Lequin, ethics is not so much a space for discussing morality or prescribed behaviours as a space for exploring meaningful departures from prevailing structures or "laws." At once a site of questioning and of recognizing the latter structures, ethics is profoundly paradoxical. The works of Ying Chen, the essay suggests, are marked by this double movement: breaking with recognized structures on the one hand and searching for a happy medium or just balance on the other. In other words, transgressing social norms is enlightening and enabling but is not a source of salvation in any absolute sense. Completely unchecked, risktaking leads to death; tempered by everyday life, it transforms and allows for the emergence of new values and ways of living.

Tenter de saisir la dimension éthique dans la littérature relève sans doute d'une démarche risquée, voire incongrue, car le mot éthique dans le contexte littéraire - oeuvres et critiques - est rarement défini et souvent utilisé de façon imprécise provoquant parfois une certaine méfiance. À tort, trop souvent synonyme du mot moral, il est associé, par plusieurs, aux prescriptions. Est-ce là l'éthique ? En suivant ce mot piège, d'un livre à l'autre, d'un critique à l'autre, l'éthique se formule pourtant autrement, toujours dans le mouvement et dans l'incertitude, plutôt que dans les certitudes. En fait, le mot informulation, dans son sens le plus dynamique, serait peut-être davantage approprié, non pas pour définir le mot éthique, mais pour mieux comprendre ses nombreux sens, complémentaires comme contradictoires.

C'est à partir d'oeuvres littéraires québécoises, d'hier et d'aujourd'hui, que je tente de suivre les traces éthiques qui se dessinent entre les lignes; les très nombreuses occurrences du mot éthique dans les oeuvres de création et de critique m'entraînant dans le désir de comprendre et de contempler base éthymologique du mot théorie. Même si je veux explorer cette notion de façon inductive en considérant les oeuvres comme agents de connaissances, l'appui philosophique me paraît également essentiel car il 
fournit des éléments de définition de l'éthique, qui à leur tour, permettent de mieux repérer le filigrane de l'éthique dans ses manifestations littéraires.

Cette incursion, à peine entamée, du côté de la philosophie, m'amène, pour l'instant, vers une approximation fonctionnelle et temporaire du mot éthique, une (in)formulation qui sous-tend les débuts de cette recherche. Il importe donc de mettre de l'avant quelques-unes de ces définitions ouvertes qui m'ont guidée dans la préparation de ce travail. Pour le philosophe Charles Taylor, selon une acception englobante, l'éthique travaille les réponses à la question du : « comment devrions-nous vivre? » (79). Le philosophe espagnol Savater, lui, explique l'éthique comme «savoirvivre » ou comme « art de vivre » (34). Elle permet d'inventer, de choisir, mais aussi de se tromper. Elle ne tranche pas les débats, elle les ouvre tous. Dans l'exercice de cet " art de vivre ", l'être humain est " à la fois artiste et objet; il est le sculpteur et le marbre » (Erich Fromm cité par Savater 50). Sibony, lui, voit la source de l'éthique dans « la cassure de la loi » (187); il pense l'éthique, non pas dans la fidélité au conforme, mais dans la mouvance; elle se forme dans la " genèse renouvelée du dire "; généreuse ou cruelle, la parole éthique "donne lieu, à de l'espace où se déploie l'imprévu du vivant.... L'éthique concerne les points de genèse de l'être parlant, plutôt que sa bonne conduite » (Sibony 188).

Les points d'intersections entre ces différentes formulations, si brèves soient-elles, sont d'une part, les lieux de questions, lieux incertains et changeants, et d'autre part, une reconnaissance implicite d'une règle, d'une loi, d'un ordre plus ou moins universel, non pas parce qu'il faut y obéir, mais parce que les questions se posent par rapport à un système et que les pratiques transgressives ou les variantes qui peuvent en résulter expriment une remise en question de ce même système, d'où le paradoxe qui sous-tend la notion de l'éthique (voir Bourdieu 141-45).

Pour entrer dans l'étude de l'éthique, je ne retiens, ici, que l'oeuvre de Ying Chen, soit ses quatre romans. Dans l'oeuvre de Chen, les personnages se situent d'une part, du côté de la non-éthique, soit l'absence de questions et de mouvement, et d'autre part, du côté de l'éthique de la dissidence. En effet, plusieurs personnages de la romancière sont confrontés à la philosophie de la moyenne ou du juste milieu et $s^{\prime}$ ensuit une règle de conformité. Dans leur entourage, la prudence est de rigueur; plutôt que de trouver leurs propres vérités, il leur faut envisager la vie sans prendre de risque. Ni les grandes passions ni les grandes joies ne sont recherchées. 
Certes, cette sagesse permet également d'éviter les grandes douleurs, mais est-ce là vivre? Dans chacun de quatre romans de Chen, cette même philosophie hante des personnages qui n'arrivent pas à se contenter d'une vie sans éclat et à se soumettre à un système de valeurs qui, en quelque sorte, entrave, pour chacun, la quête de sa propre spécificité et de ses propres valeurs. Il s'agira dans cette communication, d'une part de montrer comment joue la philosophie du juste milieu et d'autre part, de voir comment certains personnages contestent en répondant à l'appel du risque. Ils se placent alors loin des vérités toutes faites, du côté des questions. Leur risque participe d'une certaine éthique de la dissidence qui produit de nouvelles valeurs, mais aussi une conscience nouvelle du doute entraînant comparaisons, erreurs, regrets et espoirs. Bref, les jeux tensifs entre l'éthique de la prudence et l'éthique du risque seront analysés.

\section{Non-éthique ou éthique de l'extrême prudence}

Certains des personnages de Chen se situent à l'extrême pôle de la prudence et nulle question ne se pose. Dans La mémoire de l'eau, la mère de Li-Fei et $\mathrm{Ai}-\mathrm{Fu}$, sa mère nourricière, ainsi que Sheng, sa belle-mère, acceptent sans hésitation la dictature des traditions; le moindre écart face à la norme devient symbole de malheur et provoque l'angoisse, voire le rejet. Les pieds à demi atrophiés de Li-Fei sont à cet égard exemplaires. Lorsque le père ordonne l'arrêt du traitement, la mère se retire de plus en plus souvent dans sa chambre et pendant plusieurs jours, ne s'approche pas de sa fille pourtant aimée (La mémoire 22). Le mot « pied » est devenu tabou devant la petite; seul le père ose y faire référence. La servante, maman Ai$\mathrm{Fu}$, angoissée par cette transgression, ne sait plus se consoler; des années plus tard, elle demandera, sans succès, que l'on bande les pieds de Ping, sa propre petite-fille, alors que la coutume est de moins en moins pratiquée. Jamais elle n'accepte l'abandon de cette tradition, et surtout, elle se défend d'en examiner les conséquences irréversibles. De même, Sheng, la bellemère, croit que la malédiction accompagne l'entrée de Li-Fei dans sa maison et lui refuse l'accès à la cuisine, car sa seule présence pourrait empoisonner la famille. Elle voit en l'abandon de la coutume une portée apocalyptique : « À la vue des "pieds moyens" de ma grand-mère, et des pieds naturels qui abondaient dans les rues, elle croyait sincèrement que la fin du monde n'était plus très loin » (90).

Dans L'ingratitude, la mère de Yan-Zi incarne la tradition. Pour elle, rien ne doit changer. Les oiseaux en cage en témoignent. Avant la naissance de 
Yan-zi, elle comblait ses instincts maternels en s'occupant d'oiseaux que, parfois, elle punissait en les enfermant doublement : lorsqu'ils avaient trop crié, elle plaçait la cage dans la salle de bain. Elle donne d'ailleurs à sa fille un nom d'oiseau et entend la maintenir dans la cage de la tradition. Après la mort de celle-ci, elle achètera une nouvelle cage et d'autres oiseaux. Lorsque Yan-Zi lui apprend qu'elle a couché avec un homme, la mère la rejette puisque ce geste de révolte la tue, elle; le père, lui, bat sa fille incapable de comprendre sa détresse. Les compagnons de travail de Yan$\mathrm{Zi}$, eux aussi, craignent les questions de Yan-Zi et son attitude de confrontation. Toute audace de geste, même de pensée, est à bannir.

Dans Immobile, le prince en exil maîtrise son entourage; il décide pour les autres, ses épouses par exemple, et s'attend à une servilité complète de tous. Seul, lui, peut changer d'idée ou d'épouse; seul, lui, doit penser et agir. Il entend contrôler le temps et l'espace de tous ceux qui croisent son chemin. Tout son entourage, ou presque, se soumet à sa loi et vit ainsi dans l'immobilité.

Le prince incarne donc l'ordre absolu, qui, dans chacun des romans de Chen, cependant sous des formes différentes variant en intensité, domine la vie sociale et politique d'une famille, d'un pays. En découle une extrême prudence qui dépouille le sujet ou l'être de son libre arbitre. L'obéissance passive de ces personnages assujetis met en lumière la négation profonde de l'être découlant de leur conditionnement ancestral et d'une croyance profonde en la normalité. N'y a-t-il pas de meilleur esclave que l'esclave consentant? En effet, ils ne savent plus exercer leur liberté et n'osent pas douter des habitudes acquises. Ils ont peur des punitions, voire du rejet. Ils ne savent plus s'interroger sur le pourquoi des choses. Relégués au rang d'automates, ils se contentent de perpétuer fidèlement un mode de vie, leur esprit de doute s'étant complètement atrophié. Puisque l'éthique, selon plusieurs philosophes, prend racine dans une aire de questions, la notion de non-éthique appliquée à ses personnages qui n'agissent qu'à l'intérieur d'une morale imposée, soit un ensemble de comportements et de normes accepté comme nécessairement valable, permet de mieux saisir l'ampleur de leur conditionnement, en fait de leur déshumanisation.

\section{L'éthique du juste milieu}

D'autres personnages de Ying Chen ne sont pas aussi robotisés et osent entrer dans la zone des questions, toufefois en minimisant le risque. L'arrière-grand-père, dans La mémoire de l'eau, illustre cette philosophie de 
la moyenne. Il aimait " tout ce qui était moyen : richesse moyenne, intelligence moyenne, loyauté moyenne, beauté moyenne, taille moyenne... Même pour le riz, il préférait celui de longueur moyenne » (21). En politique, par exemple, il tente simultanément de plaire aux royalistes et aux révolutionnaires. Il comprend que le temps est aux changements, mais ne sachant trop comment les événements se dérouleront, il se place au milieu. C'est, pour lui, la position la plus stable. Il se détache des traditions plus facilement que sa femme d'où l'interruption du rapetissement des pieds de sa fille, non pas parce qu'il associe cette opération à de la mutilation, mais plutôt, pour que les pieds de l'enfant soient de taille moyenne. Wei-Po, le mari de Lie-Fei, partage cette éthique du juste milieu qu'il adapte aux événements marquant sa génération. Certes, il a osé épouser, contre la volonté de sa mère, une femme à pieds moyens; ce geste, en partie audacieux, relève aussi de sa compréhension des changements politiques, la tendance étant à l'abandon des traditions Il ne fait rien cependant pour protéger cette femme choisie, du courroux de sa mère qui la rejette. Son goût du risque demeure sous contrôle. Ainsi lorsqu'il déplace sa famille vers Shanghai, c'est davantage pour les affaires que par amour pour sa femme. Il n'a pas pour cette raison à affronter sa mère. Lie-Fei, ellemême, observe et jauge la société dans laquelle elle vit; par exemple, elle sent dans l'air « un goût de cadavre » (45), mais elle n'ose que rarement franchir l'obstacle des traditions. Une seule fois, intervient-elle pour qu'on épargne Ping, la petite-fille de sa mère-nourricière, du rapetissement des pieds.

Ces personnages pris entre le poids de la tradition et l'ouverture vers le changement que leur apporte l'esprit de doute, analysant leurs visions du monde et de soi, marquent l'oeuvre de Ying Chen, notamment Sassa et le père de Yuan dans Les lettres chinoises, première version. Le père recommande à son fils la prudence : « prudent en route, prudent à table, prudent en paroles » (25). Il lui rappelle que changer de pays ne change pas vraiment la vie. $D^{\prime}$ un côté, le père admire le geste de rupture du fils : une rupture culturelle, sociale et politique, d'un autre côté, la sagesse ancestrale le pousse à le mettre en garde.

Dans la deuxième version, cette lettre a été retirée, comme toutes les lettres du père, puisque le fils, lui-même, constate que l'essentiel ne change pas vraiment, notamment en comparant l'absence de liberté en Chine et l'apparence de la liberté au Québec : 
J'ai voulu me libérer un peu en quittant Shanghai. Et maintenant je cherche un patron à Montréal. Je serai employé, discipliné, payé ou congédié. J'ai choisi de vivre tout cela : je me sens donc presque libre. Mais crois-tu que je le suis vraiment ici plus qu'ailleurs? (version 1, 28; version 2, 21)

Le fils, en effet, n'a pas besoin des conseils du père, car la même sagesse, à des degrés différents, l'habite encore - au fond, l'habitera toujours - et le garde du côté de la prudence. Le fils se déplace de l'aire des questions à l'éthique de la prudence : il ose quitter son pays d'origine qu'il juge trop rigide, mais dans son nouveau pays, il découvre d'autres formes de servitude. En d'autres mots, il passe d'un geste de rupture - l'exil volontaire - à une attitude de sagesse. Il sait qu'il ne peut, de façon continue, habiter la joie de la révote ou la peine de la servitude. Il oscillera plutôt d'un pôle à l'autre, pour entre les deux, apprendre à se mieux connaître et à mieux saisir le monde qu'il habite quel qu'il soit. Ni héros ni lâche, il sera surtout animé, un peu malgré lui, comme son père, par l'éthique du juste milieu avec quelques écarts, quelques pointes de mise en doute.

Sassa, la fiancée restée à Shanghai, refuse l'émigration pour les mêmes raisons. Certes, sa maladie justifie sa décision. Son doute en lien à l'infidélité de Yuan aussi. Toutefois, au fond, elle constate à rebours qu'elle a vraiment perdu son fiancé le jour de son départ (version 1, 132-33; version $2,107-08$ ) et surtout, elle n'ose risquer l'inconnu. Elle est mécontente de sa vie et de son pays, mais croit fermement que la vie ne sera pas plus satisfaisante ailleurs. Elle s'enferme dans la sagesse du renoncement plutôt que de tenter de transformer sa vie; la peine familère étant plus acceptable que la douleur encore à venir, qui pourrait être plus intense, plus profonde; autant donc rester dans le connu. Elle est exilée dans sa propre ville; c'est là son destin. Son sens critique reste intérieur et ne se manifeste pas par des gestes concrets. Ce refus de la quête du bonheur découle, dit Ying Chen, de la religion et/ou culture boudhistes qui n'accorde aucune importance au bonheur.

\section{L'éthique de la dissidence}

Tous ses personnages, pourtant différents, participent d'une même peur, peur d'une quête inutile, peur de ne pas atteindre la paix et la joie intérieures recherchées, peur paralysante pour certains, Sassa par exemple, 
peur stimulante pour d'autres, notamment son fiancé Yuan. Ce dernier apprend très vite que sa vie à Montréal sera différente et semblable, qu'il vivra souvent en état de tension entre la sagesse ancestrale qui le fibre et l'appel du risque auquel il répond de façon mesurée. $\mathrm{Da} \mathrm{Li}$, par contre, s'engage dans une quête incessante; elle doit continuer de s'enfuir, aller ailleurs chercher la sérénité; elle aussi est tiraillée par le croisement de deux systèmes de valeurs : " Nous avons eu beau quitter cette terre, l'es-prit de Maître Con nous a suivis jusqu'ici, écrasant notre simple bonheur et nous compliquant la vie " (version 2, 129). Il est intéressant de noter que dans $\mathrm{La}$ mémoire de l'eau, le premier roman de Chen, la narratrice, la jeune femme de la famille, quitte famille et pays, par refus de la philosophie du juste milieu qui protège des grandes peines mais aussi éloigne les grandes joies. Elle veut entrer dans le risque; elle accepte à l'avance de se tromper; elle veut choisir sa propre façon de vivre et son propre système de valeurs. La seule mise en garde en lien à sa dissidence, car son départ est un geste de refus et de révolte, est l'absence d'un sentiment fort de libération : "Ainsi, à l'aéroport de New York, je n'eus pas le sentiment de soulagement que j'avais attendu depuis des années » (La mémoire 133). L'odeur de l'eau, lui disait la grandmère, n'était-elle pas " partout la même » (133). Le roman se termine sur ce retour involontaire à la prudence ancestrale comme si la révolte portait en elle ce germe d'auto-contrôle et d'auto-critique empêchant l'être révolté de se jeter à corps perdu dans une rupture irréversible, voire une autodesctruction potentielle.

Le deuxième roman met en mots la suite de cette révolte moins radicale que dans son anticipation rêvée. En effet, Yuan et $\mathrm{Da}$ Li qui ont quitté le pays pour des raisons de dissidence plus intérieure que politique, car la libération et l'accomplissment personnels en étaient les buts réels, se rendent à l'évidence qu'ils portent en eux leur culture d'origine, l'immigration n'effaçant pas le passé. Leur avenir incertain participera d'un travail d'entretissage de cultures et de valeurs que Siemerling nomme ethnogénèse, c'est-à-dire l'émergence d'une identité culturelle qui participe de la culture d'origine, mais aussi s'en différencie de façon marquée. Ils restent dans l'aire des questions constamment appelées à construire un nouvel art de vivre, toutefois sous le signe de la prudence.

Dans L'ingratitude, la dissidence est d'une tout autre nature. Yan-Zi, la jeune femme contestaire, incarne la révolte absolue. Elle ne sent que l'oppression, tant familaile que sociale. La mère devient la cible privilégiée de sa colère; elle ne perçoit que la servitude et l'autorité de cette dernière. 
Narcissique, Yan-Zi ne jauge que son propre désarroi. Elle remet tout en question, de façon effrénée, mais toujours par rapport à elle-même et à sa place, ou son manque de place, dans la société et dans la famille. En l'absence d'interlocuteur avec qui elle pourrait creuser ses questions et peut-être faire le point quant aux réponses possibles, les questions la grugent de l'intérieur et la poussent vers une révolte de plus en plus intense et de plus en plus mal ciblée. Elle arrive à se persuader que sa mère est la seule responsable de son mal de vivre. Elle ne voit pas, par exemple, que celle-ci est aussi victime de la tradition. Elle veut donc tuer sa mère pour ainsi détruire l'oppression. En effet, son suicide se veut un matricide, mais puisque la mère continue de survivre, le suicide participe de l'échec. Avant que son âme ne s'estompe tout à fait, elle se rend compte qu'il lui aurait fallu provoquer, à l'intérieur de l'espace maternel, la complicité féconde tant recherchée. Elle appelle alors sa mère, mais inutilement.

La dissidence de Yan-Zi, plus radicale et plus exaltée que celle de la narratrice de La mémoire de l'eau et celle de Yuan et $\mathrm{Da}$ Li dans Les lettres chinoises, fait table rase des valeurs du milieu. Pour échapper à l'étau social qui l'étouffe, elle s'engage dans le refus absolu, la mort. Contrairement aux deux premiers romans, où les personnages dissidents sont conscients de la sagesse ancestrale qu'ils veulent dépasser mais qu'ils savent présente, la révolte de Yan-Zi ne connaît aucun frein et elle perd ainsi tout sens critique, tout esprit de doute qui, pourtant, ont été à l'origine de sa révolte. Elle risque tout et atteint la mort plutôt qu'un nouvel art de vivre.

Dans Immobile, la narratrice vit entre deux époques. Alors que dans le temps présent, elle s'enferme dans l'immobilité, incapable de dépasser la culpabilité éprouvée dans une autre vie, puisqu'alors, son insoumission a entraîné la mort de S..., son esclave et amant. Pour se venger de l'indifférence de son mari, un prince en exil, de la solitude forcée qu'il lui impose, pour retrouver sa vie, en effet, elle séduit S... Poussée par une pulsion de révolte, cette troisième épouse du prince s'affirme, remet en question son isolement et tente de transformer le cadre de sa vie; elle n'accepte pas les ordres du prince. Cependant, lorsque l'esclave sera condamné à mort et que plus tard, le prince recevra l'ordre de mourir, elle choisira la mort, car la blessure de la mort de S... « ne se refermait pas. Elle laissait écouler les substances vitales. Je sentais pousser en moi une haïssable indifférence qui me faisait ressembler à S... J'allais jusqu'à éprouver dans le renoncement une indécente sensation de bien-être " (Immobile 139). Cette femme renonce au combat et plonge, à répétitions, 
dans sa mémoire pour essayer de comprendre l'origine de sa révolte, son attirance pour S..., sa culpabilité. Dans la vie moderne, une voyante lui rappelle que « Là où l'on demeure, c'est ainsi qu' on s'approche.... Inutile de trop s'agiter, trop chercher... » (149). La narratrice revoit alors, encore une fois sa vie antérieure et en arrive à penser qu'alors, en voulant se sauver, elle a «pris une mauvaise direction » (149). Elle devient malade et refuse de bouger. Elle n'a plus aucune envie et le roman se termine sur son immobilité. Elle attend un camion qui la prendra ou ne la prendra pas. « Tout sera alors fini. Tout recommencera " (156). Encore une fois, la révolte pourtant très grande du « je » est remise en question. L'appel du risque ne peut qu'être passager; la révolte poussée à son paroxysme est à éviter.

Dans l'oeuvre de Ying Chen, l'appel du risque permet d'avancer et d'apprendre mais ne tend pas vers le sauvetage absolu. Non freiné, cet appel conduit à la mort ou à l'auto-destruction; tempéré par les réalités de la vie quotidienne, il transforme l'être, plus ou moins en profondeur, et lui facilite l'ancrage dans sa propre vision du monde et de soi, une vision marquée à la fois par la mémoire - parfois par la nostalgie du passé - et par une indéniable soif de compréhension, et peut-être, d'accomplissement intime. Elle craint autant, semble-t-il, la révolte déchaînée que la rigidité incrustée. Ses personnages dissidents, qui vivent à la suite de leur rupture, délogent, défixent, mais leurs rêves excèdent leurs réalisations. Entre la révolte rêvée et le geste, s'installe une prudence qui dessaisit, mais aussi ressaisit. Ils prennent appui sur leurs questions et leur désordre pour rebondir dans le concret sans trop savoir où les amènera leur écart, car au fond, leur dissidence relève d'un écart toujours tensif. La narratrice de $L a$ mémoire de l'eau, Yuan dans Les lettres chinoises et la narratrice dans Immobile habitent un lieu de questions où, au jour le jour, ils se fabriquent un art de vivre encore imprévu. Ils répondent à l'appel du risque tout en craignant de s'y perdre. « Inutile de trop s'agiter ».

\section{Ouvrages cités}

Bourdieu, Pierre. Practical Reason. Traduit du français. Cambridge : Polity, 1998.

Chen, Ying. La mémoire de l'eau. Montréal : Leméac, 1992.

---. Les lettres chinoises. Montréal : Leméac, 1993. $2^{\mathrm{e}}$ édition corrigée. Leméac/Acte Sud, « collection Babel ». 1998.

--- L'ingratitude. Montréal: Leméac/Actes Sud, 1995. 
---. Immobile. Montréal : Boréal, 1998.

Savater, Fernando. Éthique à l'usage de mon fils. Paris : Seuil, 1994.

Sibony, Daniel. Jouissance du dire. Nouveaux essais sur une transmission d'inconscient. Paris: Grasset, 1985.

Siermerling Winfried. "Writing Ethnicity: Introduction ». Essays in Canadian Writing 57 (1995): 1-32.

Taylor, Charles. Les sources du moi. La formation de l'identité moderne. Trad. Charlotte Melançon. Montréal : Boréal, 1998. 